\title{
DISTANCE SETS IN METRIC SPACES
}

BY

\section{M. KELLY AND E. A. NORDHAUS}

1. Introduction. In 1920 Steinhaus $[10]\left({ }^{1}\right)$ observed that the distance set of a linear set of positive Lebesgue measure contains an interval with end point zero (an initial interval). Subsequently considerable attention was devoted to this and related ideas by Sierpinski [9], Ruziewicz [8], and others. S. Piccard in 1940 gathered together many of these results and carried out an extensive investigation of her own concerning distance sets of euclidean subsets, particularly linear subsets, in a tract entitled Sur les ensembles de distances (des ensembles de points d'un espace euclidien) [7].

Two principal problems suggest themselves in such investigations. First, what can be said of the distance set of a given space, and secondly, what can be said of the possible realizations, in a specified class of distance spaces, of a given distance set (that is, of a set of non-negative numbers including zero). The writers mentioned above have been principally concerned with the first problem, although Miss Piccard devotes some space to the second. In [4] efforts were made to improve and extend some of her results concerning the latter problem. By way of completing a survey of the literature known to us, we observe that Besicovitch and Miller [1] have examined the influence of positive Carathéodory linear measure on distance sets of subsets of $E_{2}$, while Coxeter and Todd [2] have considered the possibility of realizing finite distance sets in spaces with indefinite metrics. Numerous papers of Erdös, for example [3], contain isolated but interesting pertinent results.

In this paper we continue, in general, the investigations begun in [4]. Specifically, $\$ 2$ bridges an obvious gap in [4], $\$ 3$ extends and improves more of [7]. In $\$ \S 4$ and 5 we analyze further the concepts of rigid, proper, and irreducible $n$-sets introduced in [4], and in $\$ 6$ we consider briefly distance problems in complex and indefinite spaces.

2. Separable metric spaces. If $A$ and $B$ are two subsets of a distance space, we denote by $D(A, B)$ the set of distances $\delta(x, y)$ with $x \in A$ and $y \in B$ and by $D(A)$ the set of distances $\delta(x, y)$ with $x \in A$ and $y \in A$. Distances in the first five sections of this paper are non-negative real numbers and spaces are metric unless otherwise indicated. It follows from the definitions that

2.1. $A \subset B$ implies $D(A) \subset D(B)$ and

2.2. $A=\sum_{\alpha} A_{\alpha}$ implies $D(A)=\sum_{\alpha} D\left(A_{\alpha}\right)+\sum_{\alpha, \beta} D\left(A_{\alpha}, A_{\beta}\right)$.

Any set of distinct non-negative numbers including zero is an $n$-set, a distance set a $d$-set. If an $n$-set is a $d$-set for some subset of a specified space

Received by the editors April 20, 1951.

(1) Numbers in brackets refer to the bibliography at the end of this paper. 
or class of spaces, it is said to be realizable in that space or class of spaces.

In [4] the following results, among others, were established.

Theorem I. Any $n$-set is the $d$-set for some metric space.

THEOREM II. There exist $n$-sets which are not $d$-sets for any separable metric spaces, for example, uncountable $n$-sets with the positive numbers bounded away from zero.

TheOREM III. Any countable $n$-set is the $d$-set for a subset of Hilbert space.

THEOREM IV. There exist countable $n$-sets which are not $d$-sets for any set in any euclidean space, for example, $0,1,3,7, \cdots, 2^{n-1}-1, \cdots$.

TheOREM V. Any set of $n+1$ non-negative numbers including zero is realizable in $E_{n}$.

THEOREM VI. There exist sets of $n+2$ non-negative numbers including zero not realizable in $E_{n}$, for example, $0,1,3, \cdots, 2^{n+1}-1$.

The obvious lacuna in the above program occurs between Theorem II and Theorem III. We plug this gap with the following theorem.

THEOREM 2.1. In order that a nondenumerable $n$-set $N$ be the $d$-set for some separable metric space, it is necessary and sufficient that zero be an accumulation element of $N$.

It is convenient to lead up to the proof of this theorem with a sequence of lemmas.

LEMмA 2.1(2). If $\left\{x_{i}\right\}$ is a monotone decreasing null sequence of positive numbers, and $N$ an $n$-set on the interval $[0,1]$ whose elements are expressed in the dyadic scale, then if $N$ is metrized by defining the distance between two elements which differ first in the ith place as $x_{i}$, and as zero if two elements coincide, the resulting space is separable metric.

Proof. This is only a slight modification of the usual Baire metric. If all the real numbers on the interval $[0,1]$ be thus metrized, with the usual agreements concerning unique representation, the elements which contain only zeros from some index on may be taken as a countable basis for the space. $N$, being a subset of this space, is separable.

The space has all its triangles isosceles with legs at least as long as the base. Such spaces are sometimes referred to as nonarchimedean [6].

LEMMA 2.2. If $X=\left\{x_{i}\right\}$ is a monotone nonincreasing null sequence of positive numbers, a monotone nonincreasing sequence $Y=\left\{y_{i}\right\}$ exists, $y_{i} \in X$, with $\sum_{i=1}^{\infty} y_{i}=k$, $k$ an arbitrarily prescribed positive number, and such that $\sum_{i=r}^{\infty} y_{i}$ $\geqq y_{r-1}$.

(2) We are indebted to Professor Roy Utz for a timely suggestion in this connection. 
Proof. Select $w_{0}<k / 4$ so that $k / 2 \leqq n_{0} w_{0} \leqq 3 k / 4, n_{0}$ a positive integer. Calling $k-n_{0} w_{0}=k_{1}$, select $w_{1} \leqq w_{0} / 4$ so that $k_{1} / 2 \leqq n_{1} w_{1} \leqq 3 k_{1} / 4$. Continuing in this fashion we obtain a sequence $n_{i} w_{i}$ with $\sum_{i=0}^{\infty} n_{i} w_{i}=k$. The sequence $\left\{y_{i}\right\}$ where $y_{i}=w_{0}\left(1 \leqq i \leqq n_{0}\right), y_{i}=w_{1}\left(n_{0}<i \leqq n_{0}+n_{1}\right)$, and so on, is the desired sequence. Each $w_{i}$ is of course chosen from the sequence $\left\{x_{i}\right\}$.

Lемма 2.3. If the usual euclidean metric on the interval $[0,1]$ be transformed by introducing a modified Baire metric as described in Lemma 2.1, where, in the sequence $\left\{x_{i}\right\}, x_{i} \geqq 1 / i$, then distances are never decreased.

Proof. Since the usual Baire metric has this property, it follows that this modified Baire metric will also.

LEMma 2.4. Given a subinterval of length $x_{1}$ of the interval $[0,1]$ (with the usual euclidean metric) and a monotone decreasing null sequence $\left\{x_{i}\right\}$ $=\left(x_{1}, x_{2}, \cdots\right)$ of positive numbers, a modified Baire metric may be introduced into this interval which preserves the length of the interval and decreases the length of no distance, while the $d$-set is a subset of $\left\{x_{i}\right\}$.

Proof. Let $k_{1}$ be the first integer such that $x_{2}>1 / k_{1}$. If two numbers of the given interval differ first in the $i$ th place where $i<k_{1}$, define the distance between them to be $x_{1}$. If $k_{2}$ is the first integer such that $x_{3}>1 / k_{2}$ and two numbers differ first in the $i$ th place where $k_{1} \leqq i<k_{2}$, define their distance as $x_{2}$. Continuing in this way we generate a modified Baire space in which none of the distances is less than the corresponding euclidean distance.

Proof of Theorem 2.1. The necessity is obvious. Let $\alpha_{1}$ be a number less than unity which belongs to $N$. Select a monotone decreasing null sequence $\left\{\alpha_{i}^{\prime}\right\}$ from $N$. By Lemma 2.2 we may select a monotone nonincreasing sequence $\left\{\beta_{i}\right\}$ with

$$
\beta_{i} \in\left\{\alpha_{i}{ }^{\prime}\right\}
$$$$
\text { (2) } \quad \sum \beta_{i=1}^{\infty}=\alpha_{1} \text {, }
$$$$
\text { and }
$$$$
\text { (3) } \quad \sum_{i=r}^{\infty} \beta_{i} \geqq \beta_{r-1} \text {; }
$$

the numbers $\alpha_{r}=\sum_{i=r}^{\infty} \beta_{i}$ define a set of intervals $\left(\alpha_{i+1}, \alpha_{i}\right]$ open on the left and closed on the right. Finally the numbers $\alpha_{i}^{*}=\alpha_{1}+(i-1) \beta_{1}, i>1$, define a set of intervals $\left(\alpha_{i}^{*}, \alpha_{i+1}^{*}\right]$ open on the left and closed on the right.

Having thus partitioned the set of all non-negative numbers, we are prepared to introduce a metric into the set $N$ which will make $N$ a separable metric space with distance set $N$. Into each of the intervals to the left of $\alpha_{1}$ introduce a modified Baire metric satisfying the conditions of Lemma 2.4 where the elements of $\left\{x_{i}\right\}$ are selected from $N$. The intervals to the right of $\alpha_{1}$ are all the same length and in these we introduce a modified Baire metric making each of these intervals congruent to the interval $\left(\alpha_{2}, \alpha_{1}\right]$.

If $u$ and $v$ are elements of $N$ in the same interval, use the modified Baire metric of that interval as distance. If $u$ and $v$ are elements of $N$ not in the same interval, define $\delta(u, v)=\max (u, v)$. The triangle inequality is clearly 
satisfied in the cases where $u, v$, and $w$ are points in three different intervals and where $u, v$, and $w$ are in the same interval. If $u$ and $v$ are elements of $N$ in interval $\left(\alpha_{i+1}, \alpha_{i}\right]$, and $w$ an element of $\left(\alpha_{j+1}, \alpha_{j}\right]$ where $j<i$, then $\delta(u, w)$ $=\delta(v, w)=w$ and, since the metric was introduced so as to satisfy the conditions of Lemma $2.4, \delta(u, v) \leqq l_{i}$ where $l_{i}$ is the length of the interval in which $u$ and $v$ occur. But $w$ is clearly greater than $l_{i}$ and $\delta(u, w)+\delta(v, w)>\delta(u, v)$. It is obvious that $\delta(u, w)+\delta(u, v) \geqq \delta(v, w)$ and $\delta(v, w)+\delta(u, v) \geqq \delta(u, w)$. If on the other hand $j>i$, then $\delta(u, w)=u, \delta(v, w)=v, \delta(u, v) \leqq l_{i} \leqq \alpha_{i+1}$ by condition (3) above. But $u>\alpha_{i+1}$ and so $\delta(u, w)+\delta(v, w)>\delta(u, v)$. Finally since $\delta(u, v) \geqq|u-v|$, then $\delta(u, w)+\delta(u, v) \geqq \delta(v, w)$ and $\delta(v, w)+\delta(u, v) \geqq \delta(u, w)$.

The space is thus metric and, since each interval has a countable basis, the space has likewise. We observe finally that the space is complete.

Definition 2.1. An interval on the non-negative real axis with end point zero is called an initial interval. The interval includes zero and may or may not be closed on the right.

REMARK 2.1. If $N$ contains an initial interval, the proof of Theorem 2.1 is considerably easier. In such a case, partition the positive numbers into intervals having a length less than the length of the initial interval, these intervals being open on the left and closed on the right. Define $\delta(x, y)=|x-y|$ if $x$ and $y$ are elements of $N$ in the same interval and $\delta(x, y)=\max (x, y)$ if $x$ and $y$ are in different intervals.

THEOREM 2.2. A necessary and sufficient condition that an $n$-set $N$ be the $d$-set for some separable metric space of specified dimension $k>0$ is that $N$ contain an initial interval.

Proof. The condition is certainly necessary, for otherwise there would be arbitrarily small spheres with empty boundaries around each point and the space would be zero-dimensional (Menger-Urysohn). From Remark 2.1 it is clear that a separable metric space of dimension one can be constructed with $d$-set $N$. If, before introducing the metric described in Remark 2.1, a $k$-dimensional euclidean sphere $O$ is constructed on the first interval of the partition as diameter and the points of this sphere adjoined to $N$, with $N^{*}$ $=N+O$, a $k$-dimensional separable metric space with $d$-set $N$ may be constructed as follows. If $x$ and $y$ are elements of $N^{*}$ in the same interval, define $\delta(x, y)=|x-y|$. If $x$ and $y$ are both points of the sphere, let $\delta(x, y)$ be the usual euclidean distance. For $x$ and $y$ any other elements of $N^{*}, \delta(x, y)$ is the number of the pair furthest to the right.

We have already observed that the Baire spaces have nonarchimedean metrics. But the fact that a space has a nonarchimedean metric places no restriction on its $d$-set, as is evidenced by the following theorem.

THEOREM 2.3. Any n-set is the d-set for a nonarchimedean metric space.

Proof. Metrize the $n$-set by defining $\delta(x, y)=\max (x, y)$, and so on. 
However, calling an $n$-set $\left\{x_{i}\right\}$ (finite or infinite) isosceles if $x_{i+1}>2 x_{i}$ and $x_{1}=0$, we have the following theorem.

TheOREM 2.4. Any metric space whose $d$-set is isosceles is nonarchimedean.

Theorem $\mathrm{V}$ asserts that any $k+1$ non-negative numbers including zero are realizable in $E_{k}$, while Theorem VI assures us of the existence of sets of $k+2$ non-negative numbers including zero which cannot be realized in $E_{k}$.

There is some basis for the conjecture that any set of $k+1$ non-negative numbers including zero together with a suitably chosen $(k+2)$ th positive number would fail to be realizable in $E_{k}$. Indeed, for $k=1,2$ this is true. That this is not the case when $k>2$ is shown in the following theorem.

THEOREM 2.5. There exist $n$-sets $N$ of power $k+1$ such that any $n$-set of power $k+2$ including $N$ as a subset is realizable in $E_{k}$, for all $k>2$.

Proof. If $k=1$ and $a$ is any positive number, it is clear that there is a number $b$ such that $(0, a, b)$ is not the $n$-set for any subset of $E_{1}$. If $k=2$ an easy analysis shows that for any two positive numbers $a$ and $b, a \neq b$, there exists a number $c$ such that $(0, a, b, c)$ is not realizable in $E_{2}$.

Suppose now $k>2$ and $k$ odd. Consider the set of non-negative integer points on the $x$ axis up to $[k / 2]$ together with the point $(0, k)$. The distance set $N$ of this set of points consists of exactly $k+1$ numbers including zero. Let $r$ be any $(k+2)$ th positive number. If $r \leqq 2 k$, the set $N+r$ can be realized in $E_{3}$ by merely rotating a copy of the original set around the $x$ axis until the distance between the original point on the $y$ axis and the rotated copy of this point is $r$. If, on the other hand, $r>2 k$, the $n$-set $N+r$ may be realized in $E_{k}$ in the following fashion. Arrange the numbers $N+r$ in a monotone increasing sequence, $0<n_{1}<n_{2}<n_{3}<\cdots<n_{k}<r$. We construct a simplex $A_{0} A_{1} \cdots A_{k}$ in $E_{k}$ with $A_{i} A_{j}=n_{j}, \quad i<j<k-1 ; A_{k-1} A_{i}=n_{k-1}, \quad i<k-2$; $A_{k-1} A_{k-2}=n_{k} ; A_{k} A_{i}=r$.

For $k$ even, $k>4$, it is necessary only to omit the point $(1,0)$ in the above set. For $k=4$, examples are readily constructed.

3. Some general theorems. In this section we extend some of the theorems of [7] to more general spaces and establish other results in what might be termed the Sierpinski-Steinhaus program. The proofs in some cases are quite simple and are omitted or only briefly indicated. $\bar{P}$ denotes the set of all nonnegative real numbers.

Theorem 3.1. If $S$ is a subset of a metric space, then $D(S)$ is a continuous image of the Cartesian product $S \times S$.

THEOREM 3.2. If $S$ is a subset of a metric space, then $D(|S|) \subset|D(S)|\left(^{3}\right)$. Proof. $D(S)=f(S \times S)$, where $f$ is continuous on $S \times S$. Then $|D(S)|$

(3) For typographical reasons we use absolute value signs to denote closure; thus $|S|$ is the closure of $S$. 
$=|f(S \times S)| \supset f(|S \times S|)=f(|S| \times|S|)=D(|S|)$. Thus the adjunction of accumulation elements to a set at most adds accumulation elements to the $d$-set.

THEOREM 3.3. If in a metric space (or any space with a continuous metric) $A$ is dense in $B$, then $D(A)$ is dense in $D(B)$.

This is an immediate consequence of the relation 2.1 and Theorem 3.2.

THEOREM 3.4. If $S$ is an infinite subset of a metric space, then the power of $D(S)$ does not exceed the power of $S$.

Proof. When $S$ is infinite, the power of $S \times S$ is the same as that of $S$. Since $D(S)$ is a continuous image of $S \times S$, its power does not exceed that of $S \times S$.

TheOREM 3.5. The d-set of a closed and compact set in a metric space is closed and compact (hence bounded).

Proof. If $S$ is closed and compact, then so is $S \times S$, and the theorem follows from Theorem 3.1 and the fact that a continuous image of a closed and compact set is closed and compact.

Corollary 3.1. If $S_{1}$ and $S_{2}$ are closed and compact, then $D\left(S_{1}, S_{2}\right)$ is closed and compact.

THEOREM 3.6. If $S$ is a connected subset of a metric space, then $D(S)$ is an initial interval.

Proof. If $S$ is connected, so is $S \times S$ and any continuous image of a connected set is connected. A connected subset of $\bar{P}$ containing zero is an initial interval.

COROLlaRY 3.2. The $d$-set of a locally connected space contains an initial interval.

THEOREM 3.7. If $S_{1}$ and $S_{2}$ are connected subsets of a metric space, $D\left(S_{1}, S_{2}\right)$ is an interval.

Proof. $D\left(S_{1}, S_{2}\right)$ is a continuous real image of the connected product space $S_{1} \times S_{2}$.

Remark 3.1. From Theorem 3.6 it follows that the $d$-sets of Peano spaces, curves, complete convex metric spaces, and so on, are all initial intervals.

THEOREM 3.8. If in a metric space a set $B$ is dense in a closed compact set $A$, then $|D(B)|=D(A)$.

Proof. $B \subset A$ implies $D(B) \subset D(A)$ and $|D(B)| \subset|D(A)|=D(A)$, since $A$ is closed and compact. Furthermore, $B$ dense in $A$ implies $|B| \supset A$ or $D|B|$ $\supset D(A)$, and thus $|D(B)|=D(A)$ by Theorem 3.2.

Theorem 3.9. If $S$ has positive dimension, then $D(S)$ contains an initial interval. 
Proof. The contrary assumption implies the existence of arbitrarily small spheres around each point whose boundaries are free of points of $S$.

THEOREM 3.10. $A$ set $S$ is isolated if zero is not an accumulation element of $D(S)$.

THEOREM 3.11. If $A_{1} \supset A_{2} \supset A_{3} \supset \cdots$ is a monotone decreasing sequence of closed compact sets, then $D \prod\left(A_{i}\right)=\prod D\left(A_{i}\right)$.

Proof. The proof follows the familiar pattern of the Cantor product theorem. Easily constructed examples show that the hypotheses are necessary.

THEOREM 3.12. If $S$ is a closed, compact, and scattered subset of a separable metric space, then $D(S)$ is closed, compact, and scattered.

Proof. In a separable space, a scattered set is countable. Thus $D(S)$ is countable and, by Theorem 3.5, is closed and compact. If $D(S)$ contained a dense-in-itself subset, it would contain the closure of such a set, and thus an (uncountable) perfect set. Hence $D(S)$ is a scattered set.

THEOREM 3.13. If a subset $S$ of a metric space is Borel measurable, then $D(S)$ is analytic.

Proof. This follows from the fact that $S \times S$ is Borel measurable and a continuous transform of a Borel set is analytic.

CoRollary 3.3. If $S$ is Borel measurable, then $D(S)$ is Lebesgue measurable.

Proof. Any linear analytic set is measurable $(L)$.

Theorem 3.14. If $S$ is a projective set in a metric space, then $D(S)$ is a projective set.

Proof. The continuous transform of a projective set is projective.

Remark 3.2. If $S$ is of class $2 n$, then $D(S)$ is of class $2 n+1$, while if $S$ is of class $2 n-1$, then $D(S)$ is of class $2 n-1, n \geqq 1$.

Corollary 3.4. If $S$ is an analytic set in a metric space, then $D(S)$ is analytic.

Theorem 3.15. If $D(S)$ is a frontier set, $S$ is totally disconnected.

TheOREM 3.16. If $D(S)$ is nondense, $|S|$ is totally disconnected.

Proof. If $|S|$ contained a connected subset consisting of more than a point, then, by Theorem 3.6, $D(|S|)$ would contain an interval. Since $D(|S|)$ $C|D(S)|, D(S)$ would not be nondense.

TheOREM 3.17. If $S$ is an $F_{\sigma}$ in a locally compact separable metric space, $D(S)$ is an $F_{\sigma}$. 
Proof. Let $S=\sum_{i=1}^{\infty} F_{i}$ where $F_{i}$ is closed. For a sufficiently small radius a closed sphere having a given rational point (that is, a point of the basis) as center is compact. Thus the space is the sum of a countable collection of closed and compact sets. $F_{i}$ is thus the sum of a countable collection of closed and compact sets. It follows that $S=\sum_{i=1}^{\infty} F_{i}^{*}$ where $F_{i}^{*}$ are closed and compact. From relation $2.2, D(S)=\sum_{i=1}^{\infty} D\left(F_{i}^{*}\right)+\sum_{i, j=1}^{\infty} D\left(F_{i}^{*}, F_{j}^{*}\right)$. Since (Theorem 3.5) $D\left(F_{i}^{*}\right)$ and $D\left(F_{i}^{*}, F_{j}^{*}\right)$ are closed and compact, $D(S)$ is an $F_{\sigma}$.

CoRollary 3.5. The $d$-set of an $F_{\sigma}$ in a finitely compact metric space is an $F_{\sigma}$.

Proof. This is a consequence of the previous theorem, since a finitely compact space is separable.

REMARK 3.3. Sierpinski [9] has shown the existence in $E_{2}$ of a $G_{\delta}$ whose $d$-set is not a Borel set. It is not known whether there is a linear Borel set whose $d$-set is not a Borel set. an $F_{\sigma}$.

THEOREM 3.18. The $d$-set of an open set in a complete convex metric space is

Proof. By a fundamental theorem due to Menger, any two points of a complete convex metric space may be joined by a metric segment. It follows that open spheres are connected, and since any open set $S$ is the sum of open spheres, $D(S)$ is the union of a collection of intervals. As a consequence of the convexity, these intervals are nondegenerate, provided the space consists of more than a single point. It is easy to see that the sum of nondegenerate intervals on the line is an $F_{\sigma}$.

Definition 3.1. A set is called nearly open if its border (that is, the set minus its interior) is finite.

THEOREM 3.19. The $d$-set of an open set in a complete convex and externally convex metric space is nearly open (hence is both an $F_{\sigma}$ and $a G_{\delta}$ ).

Proof. Let $O=\sum_{\alpha} O_{\alpha}$ be an open set where $O_{\alpha}$ are open spherical neighborhoods. The $d$-set of an $O_{\alpha}$ is an initial interval open on the right. For $O_{\alpha}$ is connected and if $D\left(O_{\alpha}\right)$ were closed on the right, there would be a pair of points $p_{1}$ and $p_{2}$ of $O_{\alpha}$ such that $\delta\left(p_{1}, p_{2}\right)=\max \delta(x, y), x, y \in O_{\alpha}$. But since the space is externally convex, there would be a point $q$ such that $\delta\left(p_{1}, q\right)$ $=\delta\left(p_{1}, p_{2}\right)+\delta\left(p_{2}, q\right)$, and from the internal convexity and the transitivity of metric betweenness, it follows that there would exist a sequence of points $\left\{q_{i}\right\}$ such that $\delta\left(p_{1}, q_{i}\right)=\delta\left(p_{1}, p_{2}\right)+\delta\left(p_{2}, q_{i}\right)$ with $\lim \left\{q_{i}\right\}=p_{2}$. Thus $C\left(O_{\alpha}\right)$ would not be closed, contrary to our assumption.

In a similar fashion $D\left(O_{\alpha}, O_{\beta}\right)$ can be shown to be an open nondegenerate interval. Thus $D(O)$ is the union of a collection of open intervals and one initial interval. Such a set is an open set plus a point, and is nearly open.

REMARK 3.4. We exhibit now an open set of a complete convex metric space whose $d$-set is not a $G_{\delta}$. In the usual definition of the Cantor set on the 
interval, say 19 to 20 on the real number axis, a collection of open "middle third" intervals are removed. Denote by $H$ the union of these intervals together with their right-hand end points $\left({ }^{4}\right)$. The intervals are countable and may be denoted by $\alpha_{i}$. Suppose the length of $\alpha_{i}$ is $l_{i}$ and the end points are $x_{i}^{\prime}$, $x_{i}$ in that order. Now on the $i$ th axis in Hilbert space consider the points $A_{i}$ and $B_{i}$ at a distance $x_{i} / 2$ and $-x_{i} / 2$ respectively from the origin. The convex covering of these points may be regarded as a complete convex metric space.

Consider now the open spheres $\left(A_{i}, l_{i} / 2\right)$ and $\left(B_{i}, l_{i} / 2\right)$ in this space. The union of all such open spheres is an open set whose distance set consists of three distinct parts. First an initial interval of length $1 / 3$ open on the right. Secondly, a set of intervals obtained by considering non-diametral pairs of spheres. These intervals are all to the right of 25 on the axis. Finally, the set of intervals obtained by considering diametrically opposite spheres is readily seen to form the set $H$ on the interval 19 to 20. By Theorem 3.18 this distance set is an $F_{\sigma}$. That it is not also a $G_{\delta}$ follows from the fact that in order for a set $E$ to be both an $F_{\sigma}$ and a $G_{\delta}$ it is necessary and sufficient that $|F \cdot E| \cdot|F \cdot C(E)| \neq F$ for all non-null closed sets $F[5$, p. 207]. Taking the Cantor set on the interval 19 to 20 as $F$, the condition is seen to be violated and the set is not a $G_{\delta}$.

Corollary 3.6. The d-set of an open set in $E_{n}$, Hilbert $(H)$ space, and in fact in any Banach space is nearly open.

COROLlaRy 3.7. The d-set of hyperbolic $\left(H_{n, r}\right)$ space is nearly open.

COROLlary 3.8. The $d$-set of an open set in spherical space $\left(S_{n, r}\right)$ or elliptic space $\left(E_{n, r}\right)$ is nearly open.

Proof. Since spherical and elliptic spaces are not externally convex the argument must be modified slightly, but the fact that the only segments which cannot be "produced" are all the same length leads easily to the fact that the border of the $d$-set of an open set consists of at most two points.

REMARK 3.5. The $d$-set of a closed set in a complete convex externally convex metric space need not be an $F_{\sigma}$. (Compare with Theorem 3.17.) Consider the points of the polar curve $\rho=1+\theta$ where $\theta$ is irrational, $0<\theta<1$, together with the points of the segments joining these points to the origin. Remetrize the set as follows. Let $\delta(x, y)$ be the usual euclidean metric if $x$ and $y$ are elements of the same segment. If $x$ and $y$ are on different segments, set $\delta(x, y)=\delta(x, 0)+\delta(y, 0)$. The resulting space is metric, convex, complete, but not separable. The points on the curve and the origin constitute a discrete and hence closed set. The $d$-set of this set consists of zero, the irrational numbers between 1 and 2, and the open interval from 2 to 4 . Since the irrational numbers on an interval are not an $F_{\sigma}$, we conclude that

(4) Professor F. Herzog called our attention to this set. 
the $d$-set of a closed set in a complete convex metric space need not be an $F_{\sigma}$. By an obvious modification, the space can be made externally convex.

THEOREM 3.20. If $D(S)$ is nondense and $S$ is a subset of a complete convex metric space, then $S$ is nondense.

Proof. Since spheres are connected, $D(|S|)$ would contain an interval if $|S|$ contained a sphere. Then by Theorem 3.2, it follows that $D(S)$ would not be nondense.

THEOREM 3.21. If $D(S)$ is of the first category and $S$ is a subset of a complete convex metric space, then $S$ is of the first category.

Proof. $D(S)=\sum_{i=1}^{\infty} N_{i}$, where each $N_{i}$ is nondense. Let $M_{i}$ denote the inverse image of $N_{i}$ in $S \times S$, and $M_{i}^{*}$ and $M_{i}^{* *}$ the projections of $M_{i}$ on $S$. These starred sets form a countable covering of $S$, and if $\left|M_{i}^{*}\right|$ contains a sphere, then $\left|M_{i}\right|=\left|M_{i}^{*}\right| \times\left|M_{i}^{* *}\right|$ contains a connected subset. Since $\left|N_{i}\right|=\left|f\left(M_{i}\right)\right| \supset f\left(\left|M_{i}\right|\right),\left|N_{i}\right|$ contains an interval, contrary to assumption. $S$ is thus the countable sum of nondense sets and is of the first category.

Corollary 3.9. If $S$ is a second category subset of a complete convex metric space, then $D(S)$ is of the second category.

COROLlary 3.10. The $d$-set of $a G_{\delta}$ in a compact convex metric space is of the second category.

Proof. If $D(S)$ were of the first category, then by the previous theorem $S$ is also of the first category. However, it is known that a $G_{\delta}$ in a compact metric space is of the second category.

THEOREM 3.22. If $S$ is a subset of a Banach space, then $D(S)+D C(S)=\bar{P}$.

Proof. Suppose $n \in \bar{P}$ but $n \notin D(S)$. A sphere of radius $n$ and center at $q$, $q \in S$, has its surface lying entirely in $C(S)$. Since the surface is a connected set of diameter $2 n, D C(S)$ contains an initial interval of length $2 n$, so $n$ $\in D C(S)$.

TheOREM 3.23. If $S$ is a residual set in a Banach space, $D(S)=\bar{P}$.

Proof. Suppose $n \in \bar{P}$ but $n \notin D(S)$. A translation of $S$ a distance $n$ then carries $S$ into a subset $S^{*}$ of $C(S)$. But $C(S)$ is of the first category and $S^{*}$ is of the second, a contradiction, since no first category set can have a second category subset. Thus $D(S)=\bar{P}$.

THEOREM 3.24. If $S$ is residual at a point in a Banach space, $D(S)$ contains an initial interval.

Proof. If $S$ is residual at a point $p$, there exists a neighborhood $U$ of $p$ in which $U S$ is residual. Since $U S$ is of the second category, there exists an open subset $O$ of $U$ in which $S$ is of the second category at all of its points. 
Thus there are arbitrarily small spheres of diameter $\epsilon$ of $U$ with the same character. If $n<\epsilon$ (for suitably small $\epsilon$ ) and $n \notin D(S)$, then a translation of $S$ of norm $n$ carries the points of $S$ in this small sphere into the complement of $U S$ relative to $U$. But this complement is, by hypothesis, of the first category. This implies that for some $\epsilon$ the set $D(S)$ contains an initial interval of length $\epsilon$.

Theorem 3.25. If $S$ is a dense $G_{\delta}$ in a Banach space $B$, then $D(S)=\bar{P}$.

Proof. We may write $C(S)=\sum_{i=1}^{\infty} F_{i}$, where each $F_{i}$ is closed. Then $F_{i}$ $C C(S)$ implies $C\left(F_{i}\right) \supset S$, and since $|S|=B,\left|C\left(F_{i}\right)\right|=B$. Thus $F_{i}$ can contain no spheres, that is, $F_{i}$ is nondense. Then $C(S)$ is of the first category and $S$ is residual. The conclusion then follows from Theorem 3.23.

REMARK 3.6. In [7] it is shown that the $d$-set of a linear set dense in itself is dense in itself. In view of the rather easy extension of this theorem to $E_{2}$ and $E_{3}$, it is a little surprising that it fails to extend to higher dimensions. That it does not is evidenced by the following example.

In $E_{4}$ consider the circle $C_{1}: x_{1}^{2}+x_{2}^{2}=1, x_{3}=x_{4}=0$ and the circle $C_{2}: x_{3}^{2}$ $+x_{4}^{2}=1, x_{1}=x_{2}=0$. Since $D\left(C_{1}, C_{2}\right)=2^{1 / 2}$, it is clear that the $d$-set of the union of two small arcs of $C_{1}$ and $C_{2}$ is an initial interval together with the isolated point $2^{1 / 2}$ and is thus not dense in itself.

This same example makes it clear that the theorem is not valid in spherical 3 -space nor in elliptic 3-space. In the latter space the union of two small segments on two polar lines (which are, incidently, Clifford parallels) provides a counter-example.

Theorem 3.26. In $E_{3}$ the $d$-set of a set dense in itself is dense in itself.

Proof. Let $S$ be a subset of $E_{3}$ dense in itself and $r \in D(S)$. There exists a pair of points $p_{1}$ and $p_{2}$ of $S$ with $\delta\left(p_{1}, p_{2}\right)=r$. Consider two $\epsilon$-neighborhoods $N_{1}$ and $N_{2}$ of $p_{1}$ and $p_{2}$ respectively, for small $\epsilon$. Since $S$ is dense in itself, $N_{1}$ and $N_{2}$ contain infinitely many points of $S$. We assert that there exist points $x_{1} \in N_{1} S$ and $x_{2} \in N_{2} S$ such that $\delta\left(x_{1}, x_{2}\right) \neq r$. In the contrary case all the points of $N_{2} S$ would be at a distance $r$ from those of $S$ in $N_{1}$. This certainly cannot be the case if some four of the points in $N_{1} S$ are not coplanar, nor can it be if they are.

Corollary 3.11. In $S_{2, r}, E_{2, r}, H_{3, r}$ the $d$-set of a set dense in itself is dense in itself.

Corollary 3.12. In $E_{3}, S_{2, r}, E_{2, r}, H_{3, r}$ the $d$-set of a bounded perfect set is bounded and perfect.

Definition 3.2. A set of points of a distance space is called a metric basis for the space provided there exists no two points of the space with the same (ordered) distances to the points of the set (for example, the vertices of a proper triangle in $E_{2}$ is a metric basis for that $E_{2}$. It is not the basis for an $E_{3}$ ). 
THEOREM 3.27. If a space has a countable metric basis, the space has at most power $c$.

Proof. The power of the space cannot exceed the number of countable subsets of the real numbers.

Corollary 3.13. A separable metric space has at most power $c$.

Proof. It is readily shown that the denumerably dense set of the space is a metric basis.

THEOREM 3.28. If a space $S$ of power $p \geqq \aleph_{0}$ has a finite metric basis, the power of $D(S)$ is $p$.

Proof. Let $p_{1}$ be the power of $D(S)$ and $n$ the power of the basis. From Theorem $3.4, p \geqq p_{1}$. On the other hand, $p_{1}^{n}=p_{1} \geqq p$. So $p=p_{1}$.

Corollary 3.14. The distance set of any infinite subset of $E_{n}, S_{n, r}, H_{n, r}$, $E_{n, r}$ has the same power as the set.

REMARK 3.7. Kakutani and Erdös [3] have constructed a set in Hilbert space of power $c$ such that all distances are rational. Thus Corollary 3.14 fails to extend to Hilbert space. In [3], Erdös also shows that in $E_{k}$, any infinite set of power $m$ contains a subset of power $m$ in which no two distances are the same.

Since the Kakutani-Erdös example is not in the literature we include here a simple modification of it recently communicated to us by Professor $\mathrm{Ka}$ kutani.

Let $H$ be an infinite-dimensional separable Hilbert space, $I=[\alpha \mid 0<\alpha<1]$, and $\alpha=. a_{1}^{\alpha} a_{2}^{\alpha} a_{3}^{\alpha} \cdots$ the dyadic representation of $\alpha$. Define now a set of selector sequences $\left\{N_{\alpha}\right\}, \alpha \in I$, as follows: $N_{\alpha} \equiv\left(n_{1}^{\alpha}, n_{2}^{\alpha}, \cdots, n_{k}^{\alpha}, \cdots\right)$ where $n_{k}^{\alpha}=a_{1}^{\alpha}$ $+2 a_{2}^{\alpha}+\cdots+2^{k-1} a_{k}^{\alpha}+2^{k}, k=1,2, \cdots$. It is easily verified that (1) each $N_{\alpha}$ is a monotone strictly increasing sequence of positive integers and (2) if $\alpha \neq \beta$ then there exists a positive integer $p=p(\alpha, \beta) \geqq 1$ such that $n_{k}^{\alpha}=n_{k}^{\beta}$ for $k=1,2, \cdots, p-1$ and $n_{k}^{\alpha} \neq n_{l}^{\beta}$ for $k, l=p, p+1, \cdots$.

Let $\left\{r_{k}\right\}, k=1,2,3, \cdots$, be a sequence of positive numbers such that $\sum_{k=1}^{\infty} r_{k}^{2}<\infty$ and for which $\left[2 \sum_{k=p}^{\infty} r_{k}^{2}\right]^{1 / 2}$ is rational for $p=1,2,3, \cdots$. For each $\alpha \in I$ define $x_{n}^{\alpha}=r_{k}$ if $n=n_{k}^{\alpha}$ for some $k=1,2,3, \cdots$ and as zero otherwise. Then $x_{\alpha}=\left\{x_{n}^{\alpha}\right\}$ is for each $\alpha$ a point of $H$ and the collection of such points is the desired subset. It is easily verified that $\delta\left(x_{\alpha}, x_{\beta}\right)=\left\{\sum_{n=1}^{\infty}\left|x_{n}^{\alpha}-x_{n}^{\beta}\right|^{2}\right\}^{1 / 2}$ $=\left\{2 \sum_{k=p}^{\infty} r_{k}^{2}\right\}^{1 / 2}$ is a rational number for any $\alpha, \beta$ in $I$ where $p=p(\alpha, \beta)$ is some integer.

4. Rigid $n$-sets. We have seen that certain $n$-sets are realizable in various specified spaces, and it is natural to wonder whether any $n$-sets determine in some sense or other their associated realizations in specified spaces. A theorem of Anning and Erdös (see [4]), for example, assures us that the only possible way to realize an $n$-set consisting of an infinite number of integers in finite- 
dimensional euclidean spaces is on a line. With this in mind, it is not hard to see that the $d$-set of the set of points on the number axis consisting of positive integral powers of (say) ten can be realized in "essentially" only one way in finite-dimensional euclidean spaces. This prompts the following definition.

Definition 4.1. An $n$-set $N$ is said to be rigid relative to a class of spaces $\{S\}$ provided there is at least one realization of $N$ in $\{S\}$ and any two realizations are congruent (isometric).

THeOREM 4.1. No $n$-set is rigid relative to all metric spaces.

Proof. Case (a). Let the $n$-set be $N$ and suppose zero is not an accumulation element of $N$. Let $a$ be the g.l.b. of the positive numbers of $N$. First metrize $N$ in the now familiar fashion, $\delta(x, y)=\max (x, y)$ if $x \neq y$, and zero otherwise. Now adjoin to $N$ an element $p$ and metrize $N+p$ as follows: $\delta(x, y)=\max (x, y), x \neq p, y \neq p ; \delta(p, x)=x, x \neq 0 ; \delta(p, 0)=a$ if $a \in N ; \delta(p, 0)$ $=a+\epsilon, a+\epsilon \in N, 0<\epsilon<a$, otherwise. The two spaces have the same $d$-set but are clearly not isometric.

Case (b). Zero is an accumulation element of $N$. Metrize $N$ with $\delta(x, y)$ $=\max (x, y)$ and $N^{*}=N-0$ in the same way. These two spaces have $N$ as distance set, but are not isometric.

TheORem 4.2. No $n$-set is rigid relative to all separable metric spaces.

Proof. If the $n$-set $N$ having zero as an accumulation element be metrized as in Theorem 2.1, and then $N^{*}=N-0$ be similarly metrized with suitable adjustment of the modified Baire metric in each interval, it is quite apparent that a separable metric space $N^{*}$ may be obtained having the $d$-set $N$, but which is not congruent to $N$. If the $n$-set is countable, but does not have zero as an accumulation element, metrize it as in case (a), Theorem 4.1.

TheOREM 4.3. No $n$-set is rigid relative to all separable $k$-dimensional spaces.

Proof. Consider any $n$-set with an initial interval. By taking the first interval in the partition described in Theorem 2.2 of two different lengths, two $k$-dimensional, noncongruent, separable metric spaces with the same $d$ set may be obtained. If the $n$-set contains no initial interval, metrize it as in Theorem 4.2.

The following theorems are proved in [4].

Theorem 4.4. No countable $d$-set is rigid relative to Hilbert space.

Theorem 4.5. No finite $d$-set is rigid relative to all $E_{n}$.

On the other hand:

THEOREM 4.6. There are countable $n$-sets rigid relative to all $E_{n}$; for example, the set described in the opening paragraph of $\$ 4$. 
An $n$-set is rigid if it determines in a given class of spaces its realization uniquely up to an isometry. If an $n$-set determines its realization uniquely up to a homeomorphism, it might appropriately be called topologically rigid.

THEOREM 4.7. No n-set $N$ is topologically rigid relative to all metric spaces.

Proof. If $N$ has zero as an accumulation element, form spaces $N$ and $N^{*}$ as in case (b) of Theorem 4.1. These spaces are not homeomorphic since one contains an accumulation element and the other does not.

If the positive numbers of $N$ have a g.l.b. $a>0$, then adjoin to $N$ a set of elements $M$ whose power exceeds $c$. Metrize $N$ and $N+M$ in a manner similar to case (a) in Theorem 4.1. That is, $\delta(x, y)=\max (x, y), x, y \in N$; $\delta(p, x)=x, p \in M, x \in N, x \neq 0 ; \delta(x, y)=a, x, y \in M+0$ if $a \in N ; \delta(x, y)=a+\epsilon$, $x, y \in M+0$ with $a+\epsilon \in N, 0<\epsilon<a$, if $a \notin N$. The spaces $N$ and $M+N$ have $N$ as $d$-set but are not homeomorphic.

THEOREM 4.8. Any n-set whose positive elements are bounded away from zero is topologically rigid relative to separable metric spaces if and only if it is countably infinite.

Proof. If the $n$-set is countably infinite, any realization must have at least a countable number of points and any separable metric realization can have at most a countably infinite set of points. Furthermore, any realization must be discrete. Thus any two separable metric realizations of a countably infinite $n$-set whose positive elements are bounded away from zero are homeomorphic. That there are separable metric realizations follows from Theorem III.

If the $n$-set is finite, it cannot be metrically rigid relative even to all $E_{n}$ and thus not to all separable metric spaces, while if it is uncountable, it has no separable metric realizations.

5. Proper and irreducible $n$-sets. Theorem 4.6 prompts us to seek rigid $n$-sets realizable properly in all $E_{k}$. More specifically:

Definition 5.1. An $n$-set is called proper relative to $E_{k}$ (or properly $E_{k}$ ) if all of its realizations in euclidean spaces are in $E_{k}$, but not in $E_{k-1}$.

Thus no finite $n$-set is properly $E_{k}$ since such sets are always realizable in at least two different dimensions. The set of all positive integers and zero is properly $E_{1}$, but is not rigid relative even to $E_{1}$.

Definition 5.2. An $n$-set is called irreducibly $E_{k}$ provided it is realizable in $E_{k}$, but not in $E_{k-1}$.

Thus $0,1,3,7$ is irreducibly $E_{3}$, but not properly $E_{3}$. In [4] it was shown that an isosceles $n$-set $0, a_{1}, \cdots, a_{k}$ is irreducibly $E_{k}$. In euclidean spaces, then, rigid, proper, and irreducible are progressively weaker notions.

THEOREM 5.1. There are rigid $n$-sets, properly $E_{k}$, for all $k$.

Proof. It has already been observed that the $d$-set of the linear point set 
consisting of the origin and non-negative integral powers of ten is rigid and, further, is properly $E_{1}$. To obtain rigid sets in higher dimensions, it is necessary only to distort this set slightly. Consider, for example, the points in $E_{2}:(0,0),(1,0),(10, \epsilon),\left(10^{2}, 0\right),\left(10^{3}, 0\right), \cdots$. It is not difficult to show that the distance set of this set, for a sufficiently small $\epsilon$, is a rigid $n$-set.

Proceeding to $E_{3}$, we select a subset of points $(0,0,0),(1,0,0),(10, \epsilon, 0)$, $\left(10^{2}, 0, \epsilon\right),\left(10^{3}, 0,0\right), \cdots$. In this way rigid sets in all dimensions may be generated. As the dimension increases, the points not on the $x_{1}$-axis tend to spiral tightly around this axis.

While finite $n$-sets cannot be properly $E_{k}$ or rigid relative to all euclidean spaces, they can be irreducible and it might be wondered if for a given $k$ there are $n$-sets of all finite powers irreducibly $E_{k}$. We are not prepared to answer this question at this time, but we do assert:

THeOREM 5.2. There are $n$-sets irreducibly $E_{k}$ of arbitrarily high powers $p<\boldsymbol{\aleph}_{0}$, for all finite $k$.

We omit the proof.

The fact that the $d$-set of the integer points on the line is properly $E_{1}$ led us to conjecture that the $d$-set of the integer lattice points in $E_{k}$ might be properly $E_{k}$. However, since any integer is expressible as the sum of four squares, it is apparent that the $d$-sets of the integer lattice points in $E_{k}, k \geqq 4$, consists in every case of the square roots of all the positive integers and zero. The Anning-Erdös theorem prompted us to push the investigation a little further and we believe the following result to be of interest.

THEOREM 5.3. The n-set consisting of the square roots of all non-negative integers is irreducibly four-dimensional.

Proof. The proof that the set cannot be realized in $E_{3}$ involves an analysis of a considerable number of diophantine equations. The proof that the set cannot be realized in $E_{2}$ exhibits the essential technique, but is computationally much less involved. Accordingly, we present here a proof for this case.

Suppose $S \subset E_{2}$ existed whose $d$-set consisted of all the square roots of all the non-negative integers. Let the distance 1 , which occurs at least once, have end points at $(0,0)$ and $(1,0)$. Then an arbitrary point of $S$ has coordinates of the form $(k / 2, y)$ where $k$ is an integer, so that all points of $S$ lie on a family of parallel lines one-half unit apart. Translate to a new origin, if necessary, so that the distance $2^{1 / 2}$ occurs with end points at $(0,0)$ and $(p / 2, q)$ where $p$ is an integer. It is observed that the form of the coordinates is unaltered by this translation. Then $p^{2}+4 q^{2}=8$, and if the coordinates of any other point of $S$ be $(x, y)$, it follows from the facts that $x^{2}+y^{2}$ and $(x-p / 2)^{2}+(y-q)^{2}$ are integers that $4 q y$ is an integer, and $q^{2}$ has the possible values $1,7 / 4$, or 2 . Now since the distance $3^{1 / 2}$ must occur, the equation 
$4 q^{2} x^{2}+y^{2}=48 q^{2}$ must have a solution in integers. A simple calculation shows that this is possible only for $q^{2}=2$. Finally, since $5^{1 / 2}$ must occur, the equation $8 x^{2}+y^{2}=160$ must have a solution in integers, contrary to fact.

This disposes of the plane case and, in fact, establishes the stronger result that there is no finite or infinite point set in the plane whose distance set consists entirely of square roots of integers and contains the particular distances $1,2^{1 / 2}, 3^{1 / 2}$, and $5^{1 / 2}$.

The proof of the analogous theorem in $E_{3}$ leads to the conclusion that there is no point set in three-space whose distance set consists entirely of square roots of positive integers and contains the square roots of the seventeen integers $1,2,3,5,6,7,10,13,14,17,21,22,23,29,31,38$, and 41 .

6. Complex and indefinite spaces. If with each pair of elements $p$ and $q$ of an abstract set $K$ there is associated a complex number denoted by $(p q)^{2}$ (called the square of the distance) such that $(p q)^{2}=(q p)^{2}$ and $(p p)^{2}=0$, the resulting structure is referred to as a complex metric space. When the set $K$ consists of the $n$-tuples of complex numbers and $(x y)^{2}=\sum_{i=1}^{n}\left(x_{i}-y_{i}\right)^{2}$, the space is called complex euclidean. When $K$ consists of $n$-tuples of real numbers and $(x y)^{2}=\sum_{i=1}^{n} \epsilon_{i}\left(x_{i}-y_{i}\right)^{2}$ where of the $n$ numbers $\epsilon_{i}$ the first $r$ are +1 and the remaining $s$ are -1 , the space is called an indefinite euclidean space of dimension $n$ and denoted by $K_{r, s}$. It is clear that $K_{r, s}$ is congruent to a subset of $K_{n}, n=r+s$.

THEOREM 6.1. The $d$-set of any infinite subset of $K_{n}$ or $K_{r, s}$ has the same power as the subset.

Proof. It is well known that these spaces have finite metric bases and hence the theorem follows as in Theorem 3.28.

THEOREM 6.2. There exist countable sets of complex numbers not realizable as $d$-sets in any $K_{n}$ or $K_{r, s}$.

Proof. Consider the sequence $N=\left\{1+\epsilon / 2^{i}\right\}$. For a small $\epsilon$ these numbers are "almost equal" and hence any subset of $K_{n}$ or $K_{r, s}$ with $N$ as $d$-set would have to be "almost equilateral." But no $K_{n}$ or $K_{r, s}$ contains an almost equilateral infinite set.

THEOREM 6.3. There exist sets of $n(n+1) / 2+2$ complex numbers including zero which are not d-sets for any subsets of $K_{n}$ or $K_{r, s}$.

Proof. $n(n+1) / 2+1$ unequal, but almost equal, positive real numbers and zero constitute such a set, since any realization would have to contain at least $n+2$ points and be almost equilateral, which is impossible.

Theorem 6.4. Any set of $n(n+1) / 2+1$ complex numbers including zero is the $d$-set for a simplex in $K_{n}$.

Proof. Wald [11] has shown that any complex metric space of $n+1$ points 
is congruent to a subset of $K_{n}$.

THEOREM 6.5. Any set of $n(n+1) / 2+1$ real numbers including zero is the $d$-set for a subset of some $K_{r, s}$ of dimension $n$.

Proof. This follows from results of Wald [11] or, somewhat more specifically, from those of Coxeter and Todd [2].

\section{BIBLIOGRAPHY}

1. A. S. Besicovitch and D.S. Miller, On the set of distances between the points of a Caratheodory linearly measurable plane point set, Proc. London Math. Soc. (2) vol. 50 (1948) p. 305.

2. H. S. M. Coxeter and J. A. Todd, On points with arbitrarily assigned mutual distances, Proc. Cambridge Philos. Soc. vol. 30 (1934) pp. 1-3.

3. P. Erdös, Some remarks on set theory, Proceedings of the American Mathematical Society vol. 1 (1950) pp. 127-141.

4. L. M. Kelly, Distance sets, Canadian Journal of Mathematics vol. 3 (1951) pp. 187-194.

5. C. Kuratowski, Topologie I, Warsaw, 1933.

6. A. F. Monna, Remarques sur les metriques non-archimediennes I, Neder. Akad. Wetensch. vol. 53 (1950) pp. 470-481.

7. S. Piccard, Sur les ensembles de distances (des ensembles de points d'un espace euclidien), Mémoires de l'Université de Neuchâtel, 1939, 212 pp.

8. S. Ruziewicz, Contributions à l'étude des ensembles de distances de points, Fund. Math. vol. 7 (1925) pp. 141-143.

9. W. Sierpinski, Sur l'ensemble de distances entre les points d'un ensemble, Fund. Math. vol. 7 (1925) pp. 144-148.

10. H. Steinhaus, Sur les distances des points des ensembles de mesure positive, Fund. Math. vol. 1 (1920) pp. 93-104.

11. A. Wald, Komplexe und indefinite Räume, Ergebnisse eines Mathematischen Kolloquiums, no. 5, 1933, pp. 32-42.

Michigan State College, EAST LANSing, Mich. 